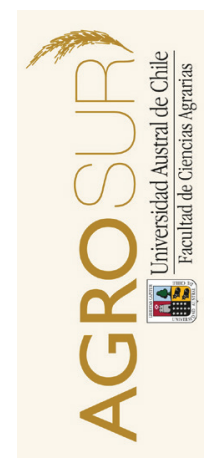

\title{
Dinámica de crecimiento y calidad nutritiva de una pradera de Lolium perenne L. sometida a diferentes frecuencias de defoliación: periodo primavera-verano
}

\author{
Growth dynamic and nutritional quality of a Lolium perenne L. pasture under \\ different defoliation frequencies: spring-summer period

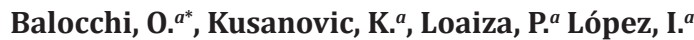 \\ ${ }^{a}$ Instituto de Producción Animal, Facultad de Ciencias Agrarias, Universidad Austral de Chile. Valdivia, Chile.
}

\begin{abstract}
ARTICLE INF O
Keywords:

Phyllocron

Perennial ryegrass

Leaf number

Original Research Article,

Animal Science

*Corresponding author:

Óscar Balocchi

E-mail address:

obalocch@uach.cl

A B S T R A C T

The study was carried out between September 2009 and January 2010 in Valdivia, Chile. Twenty seven mini-swards of Lolium perenne L. cv. Impact were established in $125 \mathrm{~L}$ containers $\left(0.181 \mathrm{~m}^{2}\right)$. A complete randomized block design was used. Nine frequencies of defoliation were evaluated, according to number of leaves per tiller (1- 1.5- 2- 2.5- 3- 3.5- 4- 4.5- 5). Measurements were taken every three days, on marked tillers, to determine leaf appearance, leaf elongation and tiller appearance. Cumulative growth was measured in each cut and forage was collected for chemical composition analysis. The results were subjected to ANOVA $(\mathrm{p}<0.05)$, Waller-Duncan means comparison test and regression analyses were made between defoliation frequency and the evaluated variables. Defoliation interval had no effect $(\mathrm{p}>0.05)$ on phyllochron. The differences in GDD varied between $56.1^{\circ}$ a $126.8^{\circ} \mathrm{C}$ /day for spring and summer, respectively. A lower defoliation frequency resulted in higher cumulative growth and leaf elongation. Tiller appearance was not affected by the different defoliation frequencies. A more frequent defoliation frequency increased $\mathrm{CP}, \mathrm{SP}, \mathrm{ME}$ and TA, whereas ADF, NDF and WSC were reduced. In conclusion, the phyllochron was independent of the defoliation frequency varying from 8 to 18.3 days, depending on the season (Spring-Summer).
\end{abstract}

\section{RESUMEN}

El estudio se realizó entre Septiembre 2009 y Enero 2010 en Valdivia, Chile. Se establecieron minipraderas monofíticas de Lolium perenne L. cv. Impact en contenedores con capacidad de $125 \mathrm{~L}$ (área de $0,181 \mathrm{~m}^{2}$ ). Se evaluaron nueve frecuencias de defoliación, basadas en el número de hojas por macollo (1- 1,5- 2- 2,5- 3- 3,5- 4- 4,5- 5 hojas/macollo), con una altura residual de $5 \mathrm{~cm}$. El diseño fue de bloques completos al azar con tres repeticiones. En cada minipradera se marcaron 3 macollos, midiendo cada tres días la aparición de hojas, elongación foliar y aparición de macollos. Del forraje cosechado se determinó crecimiento acumulado y calidad nutritiva. Los datos fueron sometidos a ANDEVA $(p<0,05)$, se utilizó el test de Waller-Duncan y se realizaron análisis de regresión entre frecuencia de defoliación y las variables evaluadas. La frecuencia de defoliación no modificó ( $p>0,05)$ el filocrono. Los grados/día requeridos para producir una hoja variaron entre $56,1^{\circ}$ a $126,8^{\circ} \mathrm{C} /$ día $^{2}$ para primavera y verano, respectivamente. Una menor frecuencia de defoliación resultó en mayor crecimiento acumulado y elongación foliar diaria, mientras que la aparición de macollos no fue modificada por la frecuencia de defoliación. El contenido de PC, PS, EM y CT disminuyó en la medida que disminuyó la frecuencia de defoliación, y el contenido de FDN, FDA y CHOS aumentó al disminuir la frecuencia de defoliación. En conclusión, el filocrono es independiente de la frecuencia de defoliación, variando de 8 a 18,3 días según época del año (primavera-verano).

Palabras clave: Filocrono, ballica perenne, número de hojas.

\section{INTRODUCCIÓN}

El manejo del pastoreo tiene como objetivo favorecer un alto rendimiento y calidad nutritiva de la pradera, una eficiente utilización del forraje producido y además lograr un alto consumo de nutrientes por parte de los animales que la pastorean. Para lograr estos objetivos, es necesario conocer la dinámica de crecimiento y calidad nutritiva de las especies forrajeras, así como el efecto que la defoliación tiene sobre estos parámetros.
El número de hojas vivas por macollo es uno de los criterios más adecuados para determinar la frecuencia de defoliación. (Fulkerson y Donaghy, 2001). La frecuencia de defoliación de una pradera puede definirse como el intervalo de tiempo entre dos cortes sucesivos. Este concepto es de suma importancia dentro del manejo del pastoreo puesto que tiene un efecto sobre la producción y la persistencia de la pradera, además con esto se logra controlar la disponibilidad y calidad del forraje ofrecido al ganado (Fulkerson y Slack, 1995). 
Estudios realizados durante los últimos años muestran que el uso de indicadores morfológicos de las especies forrajeras como base para definir los intervalos de defoliación han probado ser muy apropiados, debido a que reflejan la dinámica de crecimiento de las plantas cuando son defoliadas. Como indicador morfológico más adecuado se ha propuesto el uso del número de hojas, dado que refleja el nivel de reservas energéticas de la planta para el rebrote, además de representar de manera adecuada la calidad nutritiva del forraje a ser cosechado por los animales (Donaghy y Fulkerson, 1997, 1998).

Diversos estudios han demostrado que a menor frecuencia de defoliación, mayor es la producción de materia seca obtenida en diferentes especies forrajeras, tales como Lolium perenne, Bromus willdenowii kunth, Dactylis glomerata L., Lolium multiflorum Lam., Trifolium subterraneum L., Festuca arundinacea Schreber y Arrhenatherum elatius L. (Fulkerson y Slack, 2003; Turner et al., 2006a; Callow et al., 2005; Donaghy et al., 2008; D'Angelo et al., 2005).

La disminución en la producción de materia seca a causa de la mayor frecuencia de defoliación tendría su fundamento en el menor contenido de carbohidratos de reserva en plantas frecuentemente defoliadas. Fulkerson y Slack (1994) han demostrado la existencia de una relación lineal entre el contenido de carbohidratos de reserva y el crecimiento de las hojas al sexto día post defoliación, mientras que Turner et al. (2006c) y Donaghy et al. (2008) encontraron una relación lineal entre el contenido de carbohidratos de reserva y la producción de materia seca durante distintas etapas del desarrollo vegetativo de las especies pratenses.

Otra razón que explica la disminución en la producción de materia seca es que la frecuencia de defoliación a que son sometidas las plantas de especies forrajeras afecta en gran medida la tasa de elongación foliar (Berone et al. 2008; Donaghy y Fulkerson, 1998) Según Colabelli et al. (1998) una parte importante de la reducción de la tasa de crecimiento de una pradera, por ende el rendimiento final de esta, puede ser explicada a partir de la menor cantidad de energía lumínica interceptada, siendo esta reducida por una menor elongación de la lámina.

El filocrono corresponde al inverso de la tasa de aparición de hojas y se define como el intervalo de tiempo que existe entre la aparición de hojas sucesivas en una planta o macollo (Wilhelm y McMaster, 1995).

Diversos trabajos han demostrado que la frecuencia de defoliación no afectaría la tasa de aparición de hojas (Donaghy y Fulkerson, 1998; Velasco et al., 2007; Poff et al., 2011; Berone et al., 2008), ya que dicha tasa estaría afectada principalmente por la temperatura y la disponibilidad de agua (Wilhelm y McMaster, 1995; Fulkerson y Lowe, 2002).

Estudios han demostrado que existiría un aumento en la elongación de lámina al disminuir la frecuencia de defoliación (Donaghy y Fulkerson, 1998; Velasco et al., 2005). Según Berone et al. (2008) la tasa de elongación foliar por macollo se vio reducida inmediatamente y persistentemente en plantas defoliadas en el estado de 1,5 hojas comparadas con plantas defoliadas en el estado de 4 hojas por macollo. Velasco et al. (2005) señalan que la altura de las plantas defoliadas con mayor frecuencia se redujo considerablemente comparada a plantas defoliadas menos frecuentemente.

La frecuencia de defoliación puede afectar la aparición de macollos (Donaghy y Fulkerson, 2002; Velasco et al., 2007) pues a medida de que las defoliaciones son más frecuentes, la tasa de aparición de macollos disminuye, sin embargo, en los trabajos de Turner et al. (2006a) y Berone et al. (2008) la tasa de aparición de macollos no fue afectada por las distintas frecuencias de defoliación.

Aunque Donaghy y Fulkerson (2002), observaron que no hubo efecto de la frecuencia de defoliación en la densidad de macollos para el mes de noviembre (primavera), la dinámica de macollamiento (nuevos macollos hijos menos macollos muertos) durante todo el ensayo fue mayor en los tratamientos de defoliación infrecuente comparados a los tratamientos de defoliación frecuente, lo que reflejaría una interacción entre frecuencia de defoliación y época del año.

Hume (1991) y Donaghy y Fulkerson (1998) encontraron que altas frecuencias de defoliación estaban asociadas a un menor contenido de reservas orgánicas en las plantas, encontrando una correlación negativa entre el nivel de reservas y el tiempo en que apareció el primer macollo.

Con respecto al contenido de proteína cruda, Fulkerson y Donaghy (2001), Velasco et al. (2005), Turner et al. (2006b) y Donaghy et al. (2008) encontraron para Lolium perenne, Bromus willdenowii, Dactylis glomerata y Festuca arundinacea que a medida que aumenta la frecuencia de defoliación, aumenta el contenido de proteína en las plantas. En relación a las fracciones fibrosas, Turner et al. (2006b) señalan que al aplicar frecuencias de defoliación en el estado de 2, 3 o 4 hojas no hubo efecto sobre el contenido de fibra detergente neutro y fibra detergente ácido en las especies Dactylis glomerata y Lolium perenne, sólo existiendo un aumento de dichas fracciones en Bromus willdenowii defoliado en el estado de 4 hojas versus el estado de 2 hojas. Fulkerson y Donaghy (2001) señalan que el contenido de FDN en Lolium perenne comienza a aumentar en mayor medida luego del estado de 3,5 hojas por macollo, momento cuando empieza a senescer la hoja más vieja y comienza la elongación de tallos.

En relación al contenido de energía metabolizable, Turner et al. (2006b) indican que en Dactylis glomerata y Bromus willdenowi el contenido fue significativamente mayor en plantas defoliadas en el estado de 2 hojas 
comparado a aquellas defoliadas menos frecuentemente en el estado de 4 hojas; Fulkerson y Donaghy (2001) también señalan que la concentración de EM declina gradualmente desde el estado de 1 hoja al estado de 4 hojas.

El efecto de la frecuencia de defoliación sobre contenido de carbohidratos solubles, fue evaluado por Turner et al. (2006a) y Donaghy et al. (2008) quienes observaron que al aumentar el intervalo entre defoliaciones aumenta el contenido de carbohidratos solubles en el forraje cosechado. Fulkerson y Slack (1994) afirman que el contenido de carbohidratos solubles en el residuo, en la raíces y en las hojas de Lolium perenne tiene una relación lineal con el número de hojas por macollos. Donaghy y Fulkerson $(1997,1998)$, Donaghy y Fulkerson (2002) y Berone et al. (2008) señalan que el contenido de carbohidratos solubles en el residuo o base de las plantas, aumenta con la disminución de la frecuencia de defoliación, es decir, en plantas defoliadas con menor frecuencia aumenta el contenido de carbohidratos solubles.

Consecuentemente con lo planteado, este trabajo propuso como hipótesis que en una pradera de Lolium perenne L. la frecuencia de defoliación modifica los componentes de la dinámica de crecimiento, tales como la aparición de macollos, la elongación foliar y acumulación de materia seca. Sin embargo, la tasa de aparición de hojas no se ve afectada. Además, la frecuencia de defoliación modifica la calidad nutritiva del forraje, donde una mayor frecuencia de defoliación produce un incremento en la concentración de proteína cruda y energía metabolizable, pero una disminución en el contenido de fibra y carbohidratos solubles del forraje cosechado.

\section{MATERIAL Y MÉTODOS}

El estudio se realizó en el campus Isla Teja de la Universidad Austral de Chile, en la ciudad de Valdivia, Región de los Ríos, Chile (paralelo 3948'15,62" Latitud Sur y meridiano $73^{\circ} 15^{\prime} 13,98^{\prime \prime}$ Longitud Oeste), entre el 16 de septiembre de 2009 y el 24 de enero de 2010 (primavera temprana - verano temprano). La Figura 1 muestra la temperatura máxima, mínima y la precipitación mensual para el período experimental.

La información de temperatura y precipitaciones del período en estudio, se obtuvo del Instituto de Geociencias ubicado en el campus Isla Teja de la Universidad Austral de Chile. Con los datos de temperatura se calcularon los grados días acumulados necesarios para la aparición de una hoja, con una temperatura base de $5^{\circ} \mathrm{C}$.

Se utilizaron 27 minipraderas monofíticas de Lolium perenne cv. Impact, que fueron establecidas en febrero de 2008 (Poff et al., 2011), las cuales se encontraban en contenedores plásticos de una capacidad de $125 \mathrm{~L}$ con un área de $0,181 \mathrm{~m}^{2}$. Se establecieron 9 tratamientos, correspondientes a 9 distintas frecuencias de defoliación, basadas en el número de hojas por macollo, con

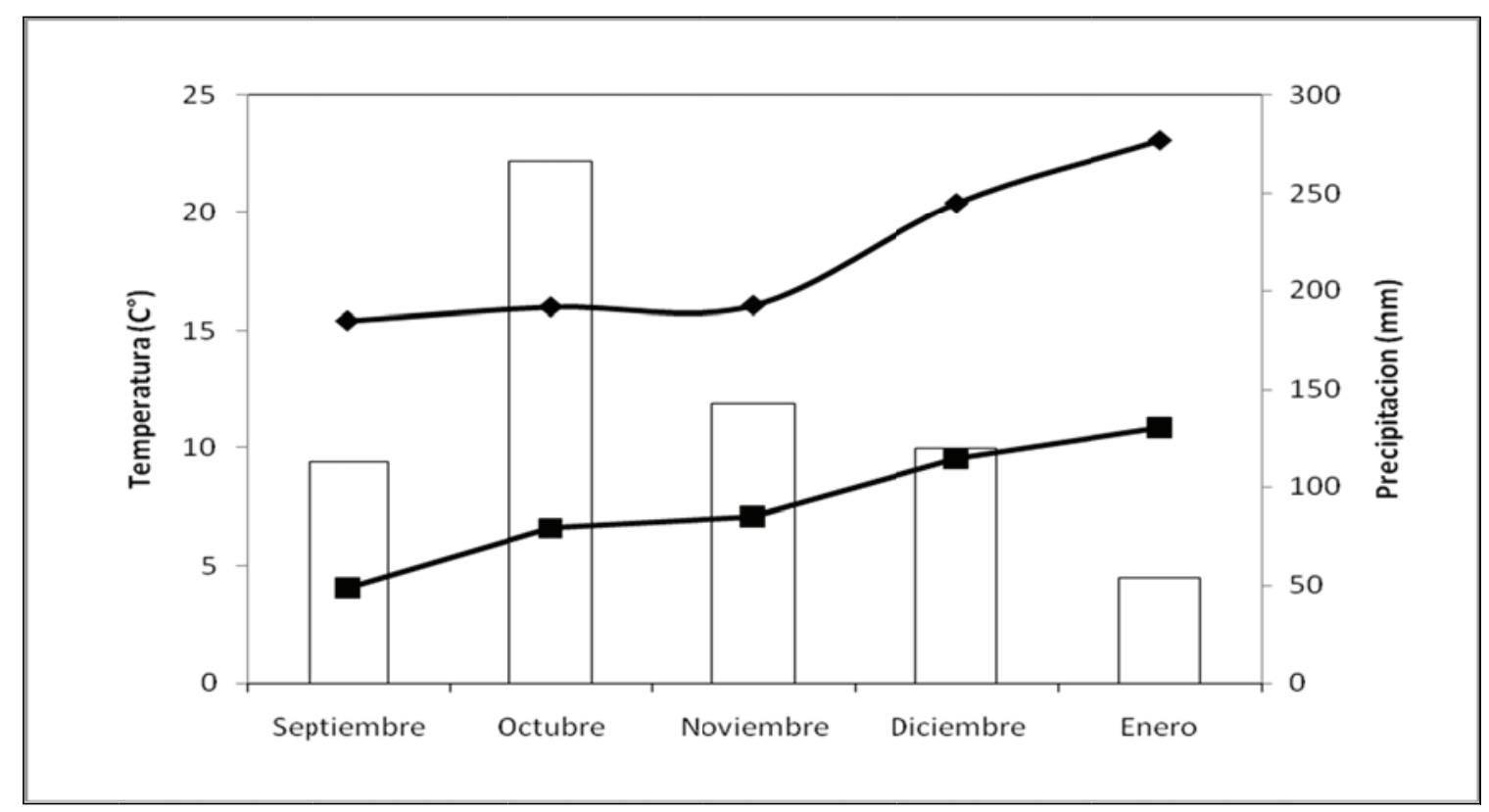

Figura 1. Media mensual de temperatura máxima ( $\downarrow$ ) y mínima ( $\bullet$ y precipitación mensual ( $\square$ ) para Valdivia, desde septiembre 2009 hasta enero 2010.

Figure 1. Monthly mean of maximum ( $\bullet$ and minimum ( $\square$ ) temperature and monthly rainfall ( $\square$ ) for Valdivia, September 2009 to January 2010. 
una altura de corte residual de $5 \mathrm{~cm}$ para todos los tratamientos, con 3 repeticiones por tratamiento.

El suelo utilizado en las minipraderas corresponde a un Andisol (Typic hapludand), perteneciente a la serie Valdivia, de la Estación Experimental Agropecuaria Austral de la Universidad Austral de Chile. El suelo se analizó en el Laboratorio de Suelos del Instituto de Ingeniería Agraria y Suelos de la Facultad de Ciencias Agrarias de la Universidad Austral de Chile (Cuadro 1). La toma de muestra fue realizada a una profundidad de $20 \mathrm{~cm}$.

El suelo fue fertilizado al inicio del experimento con un equivalente de $70 \mathrm{~kg}$ de $\mathrm{P}_{2} \mathrm{O}_{5}$ ha $^{-1}(2,75 \mathrm{~g}$ por minipradera) y $60 \mathrm{~kg}$ de $\mathrm{K}_{2} \mathrm{O} \mathrm{ha}^{-1}(1,81 \mathrm{~g}$ por minipradera), y se efectuó una fertilización nitrogenada mensual, correspondiente a $10 \mathrm{~kg}$ de $\mathrm{N} \mathrm{ha}^{-1}(0,39$ g por minipradera), aplicada en forma de urea, la que se disolvió en agua para su aplicación con una regadera. La fertilidad del suelo no se consideró como una variable en este estudio.

\section{Variables evaluadas}

Para determinar el filocrono (tasa de aparición de hojas) en cada unidad experimental se marcaron tres macollos elegidos al azar con un clip de color en su base, registrando cada tres días, en cada uno de ellos la fecha de aparición de una nueva hoja. Con esta información se calculó el tiempo térmico y los días requeridos para la aparición de una nueva hoja.

Cuadro 1. Análisis químico del suelo utilizado en el ensayo $(0-20 \mathrm{~cm})$.

Table 1. Chemical analysis of the soil used in the evaluation $(0-20 \mathrm{~cm})$

\begin{tabular}{|c|c|}
\hline Características & Nivel \\
\hline pH en agua $(1: 2,5)$ & 5,80 \\
\hline $0,01 \mathrm{M}(1: 2,5)$ & 5,30 \\
\hline Materia orgánica (\%) & 13,3 \\
\hline $\mathrm{N}$-Mineral $\left(\mathrm{N}-\mathrm{NO}_{3}+\mathrm{NH}_{4}\right)\left(\mathrm{mg} \mathrm{kg}^{-1}\right)$ & 45,5 \\
\hline Fósforo Olsen $\left(\mathrm{mg} \mathrm{kg}^{-1}\right)$ & 27,1 \\
\hline Potasio intercambiable $\left(\mathrm{mg} \mathrm{kg}^{-1}\right)$ & 98,0 \\
\hline Sodio intercambiable $\left(\mathrm{cmol}_{+} \mathrm{kg}^{-1}\right)$ & 0,22 \\
\hline Calcio intercambiable $\left(\mathrm{cmol}_{+} \mathrm{kg}^{-1}\right)$ & 5,64 \\
\hline Magnesio intercambiable $\left(\mathrm{cmol}^{+} \mathrm{kg}^{-1}\right)$ & 1,11 \\
\hline Suma de bases $\left(\mathrm{cmol}_{+} \mathrm{kg}^{-1}\right)$ & 7,22 \\
\hline Aluminio intercambiable $\left(\mathrm{cmol}_{+} \mathrm{kg}^{-1}\right)$ & 0,03 \\
\hline $\operatorname{CICE}\left(\mathrm{cmol}_{+} \mathrm{kg}^{-1}\right)$ & 7,25 \\
\hline Saturación de Al (\%) & 0,40 \\
\hline Azufre disponible $\left(\mathrm{mg} \mathrm{kg}^{-1}\right)$ & 26,1 \\
\hline
\end{tabular}

El largo de lámina y tasa de elongación foliar se midieron en los macollos marcados cada tres días. Se registró la longitud de la lámina foliar medida a partir de la lígula de la hoja anterior. Los resultados se expresaron como largo total de lámina (sumatoria de la longitud de las hojas de todos los cortes) y tasa diaria de elongación foliar ( $\mathrm{cm}$ día $\left.^{-1}\right)$.

La aparición de nuevos macollos se registró cada tres días en los macollos marcados. En el análisis de los datos se utilizó el promedio de macollos aparecidos en el periodo de evaluación en cada unidad experimental. Al momento de cumplir con el criterio de frecuencia establecido en cada tratamiento, las minipraderas fueron cortadas dejando una altura de residuo de $5 \mathrm{~cm}$, con el objetivo de medir la acumulación de fitomasa. El total del forraje obtenido en cada tratamiento fue pesado en fresco, posteriormente secado en un horno de aire forzado a $60^{\circ} \mathrm{C}$ durante 48 horas, obteniendo el contenido de materia seca.

Finalmente, se evaluó la composición nutricional del forraje, para lo cual el total de la fitomasa cosechada en cada corte fue secada y molida. Al análisis nutricional se realizó en el Laboratorio de Nutrición Animal, Instituto de Producción Animal de la Facultad de Ciencias Agrarias, Universidad Austral de Chile, utilizando espectroscopía del infrarrojo cercano (NIRS). Se obtuvieron los valores de proteína bruta (PB), proteína soluble (PS), energía metabolizable (EM), fibra detergente neutro (FDN), fibra detergente ácido (FDA), carbohidratos solubles (CHOS) y cenizas totales (CT).

\section{Diseño experimental y Análisis estadístico}

Se utilizó un diseño de bloques completos al azar con nueve tratamientos (frecuencias de defoliación) y tres bloques. Los datos obtenidos fueron sometidos a prueba de normalidad y homogeneidad de varianza, y posteriormente a un análisis de varianza (ANDEVA). Cuando se detectaron diferencias significativas $(\mathrm{p}<0,05)$ entre tratamientos, se realizó la comparación de medias con el test de Waller-Duncan. Además, se realizaron análisis de regresión entre la frecuencia de defoliación y las variables respuestas.

\section{RESULTADOS}

\section{Filocrono}

El filocrono, expresado como número de días para la aparición de una nueva hoja, no fue significativamente $(p>0,05)$ modificado por las diversas frecuencias de defoliación como se muestra en el Cuadro 2.

En el filocrono expresado como suma térmica, con base de $5^{\circ} \mathrm{C}$, para la producción de una hoja, en general, al igual que el caso anterior, no existieron diferencias significativas $(p>0,05)$ entre las frecuencias de defo- 
liación. Solo existieron diferencias estadísticamente significativas $(\mathrm{p}<0,05)$ entre los tratamientos de defoliación para la hoja cinco. Sin embargo, estas diferencias no muestran alguna tendencia lógica. Tomando en cuenta la información que se muestra en los Cuadros 2 y 3 se puede afirmar que el filocrono de Lolium perenne no se ve afectado por la frecuencia de defoliación.

Dado la falta de efecto de los tratamientos en el filocrono se realizó un análisis de varianza para comparar los días y suma térmica para la producción de una nue- va hoja. Este análisis mostró que existen diferencias altamente significativas $(\mathrm{p}<0,01)$ entre hojas. Los valores más contrastantes de suma térmica para la producción de una hoja fueron entre la hoja 1 con $63,36^{\circ} \mathrm{C}$ y la hoja 9 con $109,13^{\circ} \mathrm{C}$. Así también ocurre al comparar los días requeridos por hoja, donde 15,51 días requirió la hoja 1 y 10,33 días la hoja 10. Estos resultados muestran que la fecha o temporada de evaluación tendría un efecto significativo sobre el filocrono ya sea expresado en días o suma térmica (Cuadro 4).

Cuadro 2. Efecto de la frecuencia de defoliación sobre el filocrono, expresado en días por hoja, de Lolium perenne.

Table 2. Effect of the defoliation frequency on the phyllocron, expressed as days per leave, of Lolium perenne.

\begin{tabular}{ccccccccccc}
\hline Tratamiento & $\mathbf{h 1}$ & $\mathbf{h} \mathbf{2}$ & $\mathbf{h} \mathbf{3}$ & $\mathbf{h 4}$ & $\mathbf{h 5}$ & $\mathbf{h 6}$ & $\mathbf{h 7}$ & $\mathbf{h 8}$ & $\mathbf{h 9}$ & $\mathbf{h 1 0}$ \\
\hline $1,0 \mathrm{~h}$ & 13,33 & 12,67 & 10,67 & 12,00 & 12,67 & 13,33 & 12,00 & 9,00 & 9,67 & 9,17 \\
$1,5 \mathrm{~h}$ & 15,00 & 14,67 & 11,67 & 12,33 & 14,00 & 11,00 & 11,33 & 8,67 & 9,83 & 11,50 \\
$2,0 \mathrm{~h}$ & 13,67 & 12,33 & 11,33 & 11,00 & 10,67 & 9,67 & 11,33 & 11,00 & 11,33 & 10,33 \\
$2,5 \mathrm{~h}$ & 16,00 & 13,00 & 13,00 & 12,33 & 12,00 & 11,00 & 11,00 & 12,33 & 12,00 & 10,67 \\
$3,0 \mathrm{~h}$ & 14,67 & 13,00 & 11,33 & 12,33 & 14,33 & 11,33 & 13,00 & 10,33 & 12,00 & 12,83 \\
$3,5 \mathrm{~h}$ & 15,00 & 14,67 & 12,33 & 14,33 & 10,33 & 11,33 & 12,00 & 11,00 & 10,50 & 9,00 \\
$4,0 \mathrm{~h}$ & 18,33 & 14,67 & 15,33 & 12,83 & 13,17 & 13,33 & 13,50 & 11,50 & 12,50 & 10,00 \\
4,5 h & 16,33 & 13,67 & 14,00 & 13,33 & 12,67 & 13,33 & 10,67 & 10,00 & 11,67 & 11,50 \\
5,0 h & 17,33 & 14,00 & 15,33 & 15,33 & 13,67 & 13,33 & 10,33 & 10,67 & 12,33 & 8,00 \\
\hline Significancia & 0,3522 & 0,6350 & 0,1680 & 0,5056 & 0,1451 & 0,4524 & 0,1572 & 0,7209 & 0,5939 & 0,1211 \\
\hline
\end{tabular}

$\mathrm{h}=$ hoja

$\mathrm{p}<0,05$ Waller-Duncan

Cuadro 3. Efecto de la frecuencia de defoliación sobre el filocrono, expresado como suma térmica (base $5^{\circ} \mathrm{C}$ ) por hoja, de Lolium perenne.

Table 3. Effect of the defoliation frequency on phyllocron expressed as thermic sum (base $5^{\circ} \mathrm{C}$ ) per leave of Lolium perenne.

\begin{tabular}{|c|c|c|c|c|c|c|c|c|c|c|}
\hline Tratamiento & h1 & h2 & h3 & h4 & h5 & h6 & h7 & h8 & h9 & h10 \\
\hline $1,0 \mathrm{~h}$ & 60,47 & 72,10 & 61,23 & 68,40 & $72,33 a b c$ & 84,57 & 102,17 & 83,43 & 91,37 & 90,30 \\
\hline $1,5 \mathrm{~h}$ & 62,73 & 82,67 & 68,23 & 71,00 & $80,10 \mathrm{ab}$ & 76,73 & 93,40 & 82,43 & 98,67 & 107,93 \\
\hline $2,0 \mathrm{~h}$ & 56,13 & 69,17 & 64,33 & 64,73 & $58,83 \mathrm{c}$ & 53,17 & 85,43 & 98,57 & 104,40 & 100,57 \\
\hline $2,5 \mathrm{~h}$ & 69,03 & 78,93 & 73,80 & 71,30 & $65,43 \mathrm{bc}$ & 79,37 & 92,70 & 111,13 & 114,40 & 108,47 \\
\hline $3,0 \mathrm{~h}$ & 60,67 & 76,23 & 64,70 & 70,93 & $76,97 \mathrm{ab}$ & 78,05 & 111,23 & 99,03 & 118,07 & 126,93 \\
\hline $3,5 \mathrm{~h}$ & 57,47 & 86,80 & 69,53 & 81,87 & $57,47 \mathrm{c}$ & 79,77 & 101,13 & 108,83 & 98,97 & 90,30 \\
\hline $4,0 \mathrm{~h}$ & 71,93 & 84,37 & 89,67 & 73,57 & $89,33 \mathrm{a}$ & 93,87 & 112,50 & 100,30 & 119,00 & 95,60 \\
\hline $4,5 \mathrm{~h}$ & 70,67 & 79,83 & 82,37 & 71,53 & $79,27 \mathrm{ab}$ & 101,93 & 101,93 & 93,60 & 117,53 & 110,43 \\
\hline $5,0 \mathrm{~h}$ & 61,20 & 77,37 & 88,47 & 85,93 & $78,27 \mathrm{ab}$ & 101,80 & 92,70 & 92,53 & 119,73 & 76,43 \\
\hline Significancia & 0,420 & 0,5845 & 0,0927 & 0,7619 & 0,0042 & 0,3131 & 0,6883 & 0,7651 & 0,3008 & 0,1331 \\
\hline
\end{tabular}


Cuadro 4. Filocrono expresado en días por hoja y como suma térmica (base $5^{\circ} \mathrm{C}$ ) para las diez hojas producidas en el periodo experimental.

Table 4. Phyllocron as days per leave and as thermic sum (base $5^{\circ} \mathrm{C}$ ) for the ten leaves produced during the experimental period.

\begin{tabular}{ccl}
\hline Hoja $\mathbf{N}^{\circ}$ & $\begin{array}{c}\text { Suma térmica } \\
\text { base } \mathbf{5}^{\circ} \mathbf{C}\end{array}$ & Días hoja $^{-1}$ \\
\hline 1 & $63,36 \mathrm{e}$ & $15,51 \mathrm{a}$ \\
2 & $78,62 \mathrm{~cd}$ & $13,64 \mathrm{~b}$ \\
3 & $73,59 \mathrm{~d}$ & $12,77 \mathrm{bcd}$ \\
4 & $73,24 \mathrm{~d}$ & $12,84 \mathrm{bc}$ \\
5 & $72,82 \mathrm{~d}$ & $12,62 \mathrm{bcd}$ \\
6 & $83,28 \mathrm{c}$ & $11,94 \mathrm{cde}$ \\
7 & $99,24 \mathrm{~b}$ & $11,68 \mathrm{de}$ \\
8 & $96,63 \mathrm{~b}$ & $10,50 \mathrm{f}$ \\
9 & $109,13 \mathrm{a}$ & $11,31 \mathrm{ef}$ \\
10 & $100,77 \mathrm{ab}$ & $10,33 \mathrm{f}$ \\
\hline Significancia & 0,0001 & 0,0001
\end{tabular}

$\mathrm{p}<0,05$ Waller-Duncan

\section{Largo total de lámina, tasa de elongación foliar y aparición de macollos}

En el Cuadro 5 se observa que existen diferencias altamente significativas $(\mathrm{p}<0,01)$ en la tasa de elongación foliar (TEF, cm día ${ }^{-1}$ ) y en el largo total de lámina (cm) de los macollos de Lolium perenne por efecto de las diferentes frecuencias de defoliación.

El tratamiento 5,0 hojas registró la mayor tasa de elongación foliar $\left(0,9 \mathrm{~cm} \mathrm{día}^{-1}\right)$, no siendo estadísticamente diferente del tratamiento 3,5 hojas. Por otro lado la menor tasa de elongación foliar $\left(0,59 \mathrm{~cm} \mathrm{día}{ }^{-1}\right) \mathrm{se} \mathrm{ob-}$ servó en los tratamientos 1,0 y 1,5 hojas, sin ser estadísticamente diferentes de los tratamientos 2,0; 2,5; 3,0 y 4,0 hojas. Según el análisis de regresión la tasa de elongación foliar aumentaría en $0,0662 \mathrm{~cm}$ día $^{-1}$ al disminuir la frecuencia de defoliación en 0,5 hojas (Figura 2b).

El largo total de lámina fue mayor $(114,84 \mathrm{~cm})$ en el tratamiento 5,0 hojas, no difiriendo estadísticamente del tratamiento 3,5 hojas. El menor valor $(72,57 \mathrm{~cm})$ se observó en la frecuencia de defoliación de 1,5 hojas, no siendo diferente estadísticamente de los tratamientos 1,0, 2,0, 2,5, 3,0 y 4,0 hojas (Cuadro 5). La relación entre largo total de lámina y frecuencia de defoliación es altamente significativa $(p<0,01)$, donde la ecuación de regresión en la Figura 2a muestra que una disminución de la frecuencia de defoliación en 0,5 hojas aumentaría el largo total de lámina en $8,69 \mathrm{~cm}$.
La aparición de macollos no se vio afectada por las diferentes frecuencias de defoliación, no existiendo diferencias significativas $(p>0,05)$ en el número de macollos aparecidos en cada tratamiento, con valores que variaron entre 1,89 a 2,89 macollos aparecidos en cada macollo marcado durante el periodo de evaluación (Cuadro 5).

\section{Crecimiento acumulado}

Las diferentes frecuencias de defoliación a que fue sometida la pradera en el período de primavera-verano modificaron significativamente $(\mathrm{p}<0,05)$ el crecimiento acumulado ( $\mathrm{g} \mathrm{MS} \mathrm{m}^{-2}$ ). El Cuadro 5 muestra que el tratamiento 5,0 hojas tuvo el mayor rendimiento $(576,96 \mathrm{~g}$ $\mathrm{MS} \mathrm{m}^{-2}$ ), no siendo este estadísticamente diferente a los tratamientos 3,5; 4,0 y 4,5 hojas; cuyos rendimientos fueron 475,88 - 533,00 - 432,99 $\mathrm{g} \mathrm{MS} \mathrm{m}^{-2}$, respectivamente. El tratamiento con menor rendimiento $(351,94 \mathrm{~g}$ $\mathrm{MS} \mathrm{m}^{-2}$ ) fue el de 1,5 hojas, no siendo este estadísticamente diferente de los tratamientos 1,0; 2,0; 2,5; 3,0; 3,5 y 4,5 hojas (Cuadro 5).

El análisis de regresión muestra una relación lineal significativa $(\mathrm{p}<0,01)$ entre la frecuencia de defoliación y el crecimiento acumulado de Lolium perenne, siendo este mayor cuanto menos frecuente es la defoliación. Según la ecuación de regresión por cada 0,5 hojas de disminución en la frecuencia de defoliación, el crecimiento acumulado del período aumentaría 47,96 $\mathrm{g} \mathrm{MS} \mathrm{m}^{-2}$ (Figura 2c).

\section{Calidad nutricional del forraje}

La calidad nutricional del forraje cosechado de la pradera de Lolium perenne fue modificada de forma significativa por la frecuencia de defoliación para todas las variables evaluadas. El análisis de regresión muestra una relación altamente significativa $(\mathrm{p}<0,01)$ entre la frecuencia de defoliación y el contenido de PC del forraje de Lolium perenne, en donde una disminución de 0,5 hojas en la frecuencia de defoliación produciría también una disminución del contenido de proteína cruda en 2,57 unidades de porcentaje (Figura 3a). De igual forma se obtuvo una relación altamente significativa $(\mathrm{p}<0,01)$ entre el contenido de proteína soluble y la frecuencia de defoliación, donde una disminución de 0,5 hojas en la frecuencia de defoliación produciría una disminución de 1,23 unidades de porcentaje en el contenido de PS (Figura 3b).

La energía metabolizable (EM) del forraje cosechado varió entre 2,83 y $2,62 \mathrm{Mcal} \mathrm{kg}^{-1}$ de MS, correspondiendo estos valores a los tratamientos 1,0 y 5,0 hojas, respectivamente. La regresión entre frecuencia de defoliación y contenido de energía metabolizable del forraje es altamente significativa $(\mathrm{p}<0,01)$, observándose que al disminuir la frecuencia de defoliación en 0,5 
Cuadro 5. Efecto de la frecuencia de defoliación sobre la dinámica de crecimiento de Lolium perenne.

Table 5. Effect of the defoliation frequency on the growth dynamics of Lolium perenne.

\begin{tabular}{ccccc}
\hline Tratamiento & $\begin{array}{c}\text { Tasa elongación foliar } \\
\left(\mathrm{cm} \mathrm{día}^{-1}\right)\end{array}$ & $\begin{array}{c}\text { Largo total de lámina } \\
(\mathrm{cm})\end{array}$ & $\begin{array}{c}\text { Aparición. Macollos } \\
\left(\mathrm{N}^{\circ} \text { macollos }\right)\end{array}$ & $\begin{array}{c}\text { Crecimiento acumulado } \\
\left(\mathrm{g} \mathrm{MS} \mathrm{m}^{-2}\right)\end{array}$ \\
\hline $1,0 \mathrm{~h}$ & $0,59 \mathrm{~d}$ & $73,65 \mathrm{~d}$ & 1,89 & $367,40 \mathrm{c}$ \\
$1,5 \mathrm{~h}$ & $0,59 \mathrm{~d}$ & $72,57 \mathrm{~d}$ & 2,11 & $351,94 \mathrm{c}$ \\
$2,0 \mathrm{~h}$ & $0,65 \mathrm{~cd}$ & $81,61 \mathrm{~cd}$ & 3,00 & $376,27 \mathrm{c}$ \\
$2,5 \mathrm{~h}$ & $0,61 \mathrm{~d}$ & $77,59 \mathrm{~cd}$ & 3,45 & $416,39 \mathrm{bc}$ \\
$3,0 \mathrm{~h}$ & $0,66 \mathrm{~cd}$ & $83,18 \mathrm{~cd}$ & 1,89 & $370,43 \mathrm{c}$ \\
$3,5 \mathrm{~h}$ & $0,82 \mathrm{ab}$ & $102,56 \mathrm{ab}$ & 2,89 & $475,88 \mathrm{abc}$ \\
$4,0 \mathrm{~h}$ & $0,67 \mathrm{~cd}$ & $84,30 \mathrm{~cd}$ & 1,89 & $533,00 \mathrm{ab}$ \\
$4,5 \mathrm{~h}$ & $0,76 \mathrm{bc}$ & $94,46 \mathrm{bc}$ & 2,44 & $432,99 \mathrm{abc}$ \\
$5,0 \mathrm{~h}$ & $0,90 \mathrm{a}$ & $114,84 \mathrm{a}$ & 2,89 & $574,96 \mathrm{a}$ \\
\hline Significancia & 0,0014 & 0,0014 & 0,7733 & 0,0191 \\
\hline
\end{tabular}

$\mathrm{h}=$ hojas

$\mathrm{p}<0,05$ Waller-Duncan

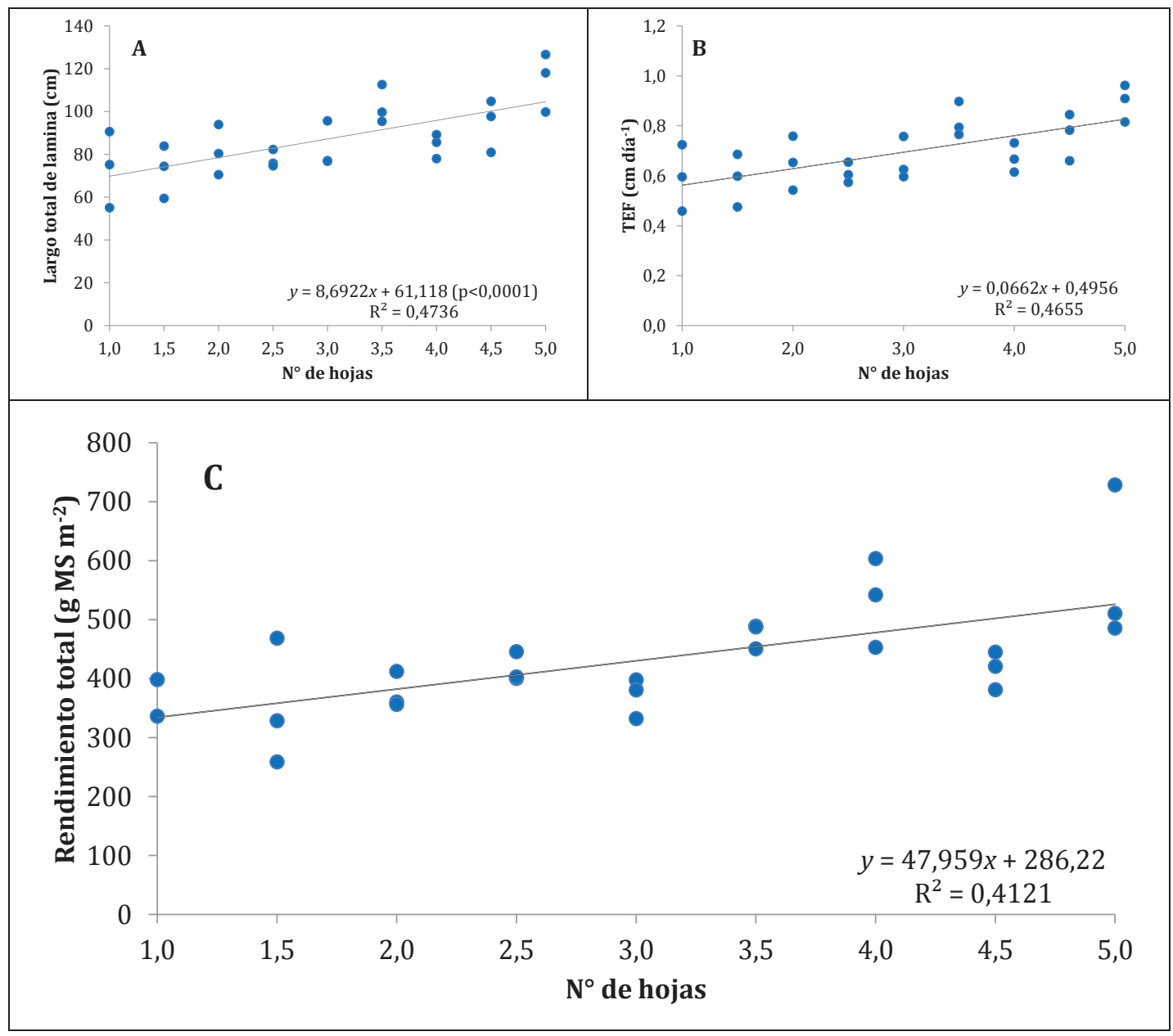

Figura 2. Relación entre la frecuencia de defoliación y largo total de lámina (a), tasa de elongación foliar (b) y crecimiento acumulado (c), en Lolium perenne.

Figure 2. Relationship between defoliation frequency and total leaf length (a), foliar elongation rate (b) and accumulated growth (c) in Lolium perenne. 


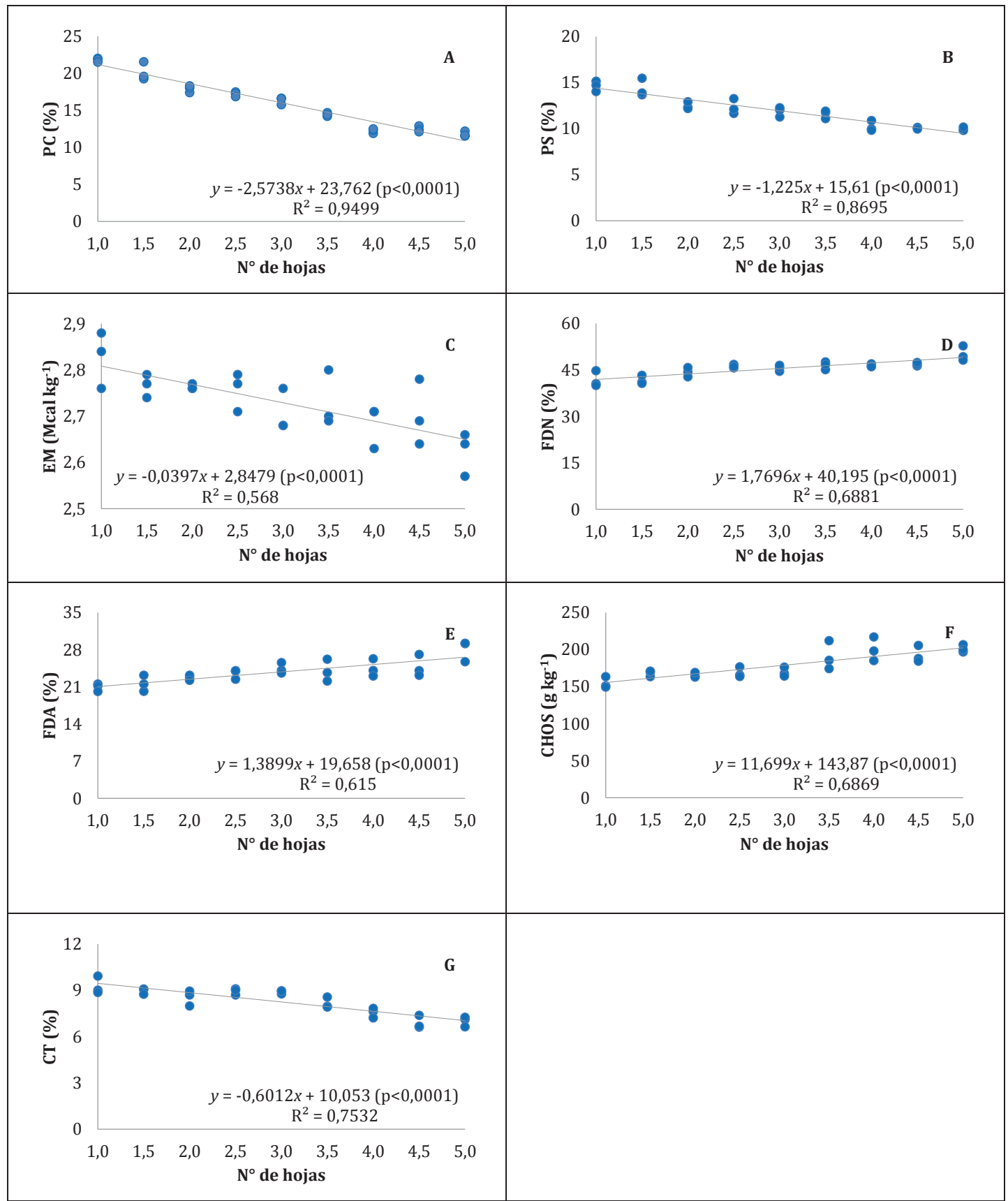

Figura 3. Efecto de la frecuencia de defoliación sobre parámetros de la calidad nutricional del forraje cosechado de Lolium perenne.

Figure 3. Effect of the defoliation frequency on nutritional quality parameters of Lolium perenne harvested forage.

hojas el contenido de EM disminuiría en 0,0397 Mcal $\mathrm{kg}^{-1}$ de MS (Figura 3c).

El contenido de FDN y FDA se relacionó significativamente $(p<0,01)$ con la frecuencia de defoliación. Según la ecuación de regresión el contenido de FDN y FDA aumentaría en 1,77 y 1,39 unidades de porcentaje, respectivamente, al disminuir la frecuencia de corte en 0,5 hojas (Figura $3 \mathrm{~d}$ y $3 \mathrm{e}$ ).
La concentración de CHOS varió entre 201,08 y $154,63 \mathrm{~g} \mathrm{~kg}^{-1}$ de MS, siendo el tratamiento 5,0 hojas el de mayor contenido y el tratamiento 1,0 hojas el de menor contenido. La concentración de CHOS se relacionó significativamente $(\mathrm{p}<0,01)$ con la frecuencia de defoliación, donde según la ecuación de regresión, al disminuir en 0,5 hojas la frecuencia de defoliación aumentaría en 11,70 $\mathrm{g} \mathrm{kg}^{-1}$ de MS la concentración de CHOS (Figura 3f). 
El contenido de cenizas totales (CT) se relacionó significativamente $(\mathrm{p}<0,01)$ con la frecuencia de defoliación, en donde una disminución de 0,5 hojas en la frecuencia de defoliación disminuiría el contenido porcentual de CT en 0,60 unidades de porcentaje (Figura $3 \mathrm{~g}$ ). El tratamiento 1,0 hojas mostró el mayor contenido $(9,27 \%)$ de CT y el tratamiento 4,5 hojas el menor contenido $(6,89 \%)$.

\section{DISCUSIÓN}

En el presente trabajo se observó que, en general, el crecimiento acumulado ( $\mathrm{g} \mathrm{MS} \mathrm{m}^{-2}$ ) de las minipraderas fue mayor en aquellas que fueron defoliadas de manera menos frecuente. Estos resultados concuerdan con lo señalado por diversos autores para especies como Lolium perenne, Bromus willdenowii, Dactylis glomerata, Lolium multiflorum, Trifolium subterraneum, Trifolium repens, Festuca arundinacea y Arrhenatherum eliatus (Donaghy y Fulkerson, 1997, 1998; Fulkerson y Slack, 2003; Velasco et al., 2005, 2007; Poff et al. 2011; Castro, 2010; Turner et al. (2006a); Callow et al., 2005; Donaghy et al., 2008; D’Angelo et al., 2005).

Una explicación para la disminución en el crecimiento acumulado cuando la defoliación es frecuente se ha relacionado con el efecto negativo de esta, sobre la tasa de elongación foliar. Donaghy y Fulkerson (1998) y Berone et al. (2008) encontraron que en plantas frecuentemente defoliadas se reducía significativamente la tasa de elongación foliar. Otro motivo del menor crecimiento acumulado de plantas frecuentemente defoliadas puede ser la significativa diferencia en biomasa radical, tasa de elongación radical e inicio del crecimiento radical, entre plantas frecuentemente defoliadas y plantas defoliadas menos frecuentemente. Estas variables no fueron evaluadas en el presente estudio, sin embargo según los estudios de Donaghy et al. (1997), Donaghy y Fulkerson (1998), Donaghy y Fulkerson (2002) y Callow et al. (2005), demuestran que la biomasa radical, la tasa de elongación radical y el inicio del crecimiento radical disminuye y se retarda, respectivamente, en plantas frecuentemente defoliadas, influyendo en el rendimiento total puesto que cualquier factor que retarde el crecimiento radical tiene un efecto indirecto sobre el crecimiento del resto de la planta, dado por la restricción en la absorción de nutrientes y agua (Davidson y Milthorpe, 1966; Donaghy y Fulkerson, 1998).

La falta de respuesta del filocrono a las distintas frecuencia de defoliación confirma parte de la hipótesis planteada y concuerda con resultados obtenidos por Velasco et al. (2007); Berone et al. (2008), Poff et al. (2011), y Castro (2010). La principal razón de la falta de respuesta a la frecuencia de defoliación es que el filocrono es dependiente principalmente de la temperatura (Fulkerson y Lowe, 2002; Wilhelm y
McMaster, 1995), observándose en este estudio que a medida que aumentaban las temperaturas, conforme el avance hacia la época de verano, disminuyeron los días necesarios para la producción de una nueva hoja.

Se observó un aumento del filocrono, expresado como suma térmica, en la medida que se avanzó de primavera a verano, con un aumento de las temperaturas diarias y un alargamiento de los días. Resultados similares fueron obtenidos por Castro (2010), en donde una menor suma térmica fue necesaria para la producción de una nueva hoja en la época de invierno en comparación a épocas de mayor temperatura y duración del día. Fulkerson y Donaghy (2001), indican una estrecha relación entre la tasa de aparición de hojas y la temperatura indicando que se necesitan de 5 a 7 días para que aparezca una hoja en la estación de primavera y de 20 a 30 días a mediados de invierno.

La tasa de elongación foliar fue modificada por las distintas frecuencias de defoliación, donde una defoliación poco frecuente (5 hojas) reportó la tasa más alta, sin embargo, cabe destacar la falta de diferencias más notorias entre los tratamientos de defoliación más frecuentes. Donaghy y Fulkerson (1998) reportan que la tasa de elongación foliar en plantas de Lolium perenne defoliadas de forma frecuente en el estado de 1 hojas se vio significativamente reducida durante los primeros 11 días de rebrote, en comparación a plantas defoliadas menos frecuente en el estado de 3 hojas. Estos autores postulan que existe una relación lineal significativa entre la tasa de elongación foliar durante los primeros 11 días de rebrote y la cantidad de carbohidratos solubles de reserva ( $\mathrm{mg} \mathrm{macollo}^{-1}$ ) en la base de los macollos antes de la defoliación. Similar resultado obtuvieron Berone et al. (2008), en Lolium perenne y Bromus stamineus, encontrando que la tasa de elongación de lámina por macollo se vio reducida inmediatamente y persistentemente en plantas defoliadas en el estado de 1,5 hojas comparadas a plantas defoliadas en el estado de 4 hojas por macollo, encontrando además que plantas defoliadas frecuentemente poseían una baja concentración de carbohidratos de reserva en la base de los macollos, al igual que en el trabajo de Donaghy y Fulkerson (1998). Estudios realizados por Castro (2011) en el mismo sitio que el presente trabajo, reportan resultados que concuerdan con los obtenidos en este estudio y otros autores como Velasco et al. (2005), quienes obtuvieron mayores alturas de plantas cuando fueron defoliadas de forma infrecuente comparadas a plantas defoliadas con mayor frecuencia.

La aparición de macollos fue una de las variables que no se vió significativamente afectada por las distintas frecuencias de defoliación a que fueron sometidas las minipraderas. Esta respuesta concuerda con los resultados obtenidos por Turner et al. (2006a) y Donaghy y Fulkerson (2002). La dinámica de maco- 
llamiento de especies forrajeras se considera que es variable e influenciada por varios factores, como son la cantidad de carbohidratos de reserva en la base de los macollos al momento de la defoliación (Hume, 1991; Donaghy y Fulkerson, 1998), la calidad de luz incidente en las yemas de crecimiento en la base de los macollos (Colabelli et al., 1998) y también la época del año (Donaghy et al., 1997).

Las distintas frecuencias de defoliación tuvieron un efecto significativo sobre las variables de calidad nutritiva del forraje cosechado, donde una mayor frecuencia incrementó el contenido de proteína (cruda y soluble) y energía metabolizable, pero disminuyó los contenidos de fibra (FDN y FDA) y carbohidratos solubles del forraje.

Los resultados obtenidos para PC concuerdan con Fulkerson y Donaghy (2001), Velasco et al. (2005) y Nave et al. (2013) en Lolium perenne; Turner et al. (2006b) y Donaghy et al. (2008) en Bromus willdenowii, Dactylis glomerata y Festuca arundinacea, respectivamente.

La relación entre frecuencia de defoliación y contenido de energía metabolizable del forraje cosechado se observó también en los trabajos de Turner et al. (2006b) y de Donaghy et al. (2008). Fulkerson y Donaghy (2001) señalan que la concentración de EM declina gradualmente desde el estado de 1 hoja al estado de 4 hojas lo que concuerda con lo obtenido en este estudio. Esto se explica principalmente como consecuencia de la disminución de la digestibilidad, la cual puede ser explicada por el aumento en la proporción de esclerénquima y tejido vascular con el incremento en el número de hojas en un macollo (Turner et al., 2006b). Velasco et al. (2005) afirman que la digestibilidad del forraje cosechado disminuye a medida que aumenta el intervalo de defoliación, esto estaría causado por el aumento en los contenidos de fibra.

En relación al contenido de fibra del forraje cosechado (FDN y FDA), Fulkerson y Donaghy (2001) señalan que en Lolium perenne comienza a aumentar en mayor medida luego del estado de 3,5 hojas por macollo, momento cuando empieza a senescer la hoja más vieja y comienza la elongación de tallos. Similares resultados obtuvieron Donaghy et al. (2008), señalando que los contenidos de FDN y FDA en Festuca arundinacea defoliada en el estado de 1 hoja fueron significativamente menores que los encontrados en plantas defoliadas con menos frecuencia $(2$ y 4 hojas). Lo anterior concuerda con los resultados obtenidos en el presente estudio, donde los contenidos de FDN y FDA alcanzaron los máximos valores en los tratamientos con intervalos de defoliación mayores. La explicación al aumento de la fibra está relacionada con la edad de la planta (número de hojas) y aumenta a una tasa influenciada por la magnitud de cambio de las concentraciones de carbohidratos estructurales de la pared celular y lignina (Donaghy et al., 2008).
Los carbohidratos solubles son producto de la fotosíntesis, su producción y posterior acumulación siguen una curva que varía a través de las fases de crecimiento (Fulkerson y Donaghy, 2001). En general para plantas forrajeras perennes el contenido de carbohidratos solubles es bajo al comienzo del período de crecimiento y alto en la etapa cercana a la madurez. Los resultados obtenidos en el presente estudio, concuerdan con los obtenidos por Turner et al. (2006a) y Donaghy et al. (2008), donde al aumentar el intervalo entre defoliaciones aumenta también el contenido de carbohidratos solubles en el forraje cosechado. Fulkerson y Slack (1994) afirman en su trabajo que el contenido de carbohidratos solubles en el residuo, en la raíces y en las hojas de Lolium perenne tiene una relación lineal con el número de hojas por macollos. Puesto que los carbohidratos solubles son producidos por la fotosíntesis, se explica que a mayor número de hojas por macollos (edad de la planta), mayor es el área para hacer fotosíntesis, por lo tanto mayor es el contenido de carbohidratos solubles en el forraje cosechado.

\section{CONCLUSIONES}

La frecuencia de defoliación no modificó el filocrono y la aparición de macollos. Sin embargo, afectó la elongación foliar diaria, el largo total de la lámina y la fitomasa producida, las que fueron mayores con defoliaciones infrecuentes. La calidad nutritiva del forraje cosechado fue significativamente modificada por la frecuencia de defoliación, aumentando la concentración de PC, PS y EM y disminuyendo la concentración de FDN, FDA y CHOS en la medida que se aumenta la frecuencia de defoliación.

\section{REFERENCIAS}

BERONE, G., LATTANZI, F., AGNUSDEI, M., BERTOLOTTI, N. 2008. Growth of individual tillers and tillering rate of Lolium perenne and Bromus stamineus subjected to two defoliation frequencies in winter in Argentina. Grass and Forage Science 63: 504-512.

CALLOW, M., MICHELL, P., BAKER, J., COCKS, P., HOUGH, G. 2005. Response of herbage regrowth and water soluble carbohydrate concentration of ryegrass species to defoliation practices when grown in mediterranean environment. Grass and Forage Science 50: 59-67.

CASTRO, J. 2010. Dinámica de crecimiento y calidad nutritiva de una pradera de Lolium perenne L. sometida a diferentes frecuencias de defoliación. Periodo otoño-invierno. Tesis Magister en Ciencias, mención Producción Animal, Universidad Austral de Chile, Valdivia, Chile 62 p.

COLABELLI, M., AGNUSDEI, M., MAZZANTI, A., LABREVEUX, M. 1998. El proceso de crecimiento y desarrollo de gramíneas forrajeras como base para el manejo de la de- 
foliación. Boletín técnico $\mathrm{N}^{\circ}$ 148. Instituto Nacional de Tecnología Agropecuaria. Buenos Aires. 15 p.

DAVIDSON, J., MILTHORPE, F. 1966. Leaf growth in Dactylis glomerata following defoliation. Annals of Botany 30: 173-184.

DONAGHY, D., FULKERSON, W. 1997. The importance of water soluble carbohydrate reserves on regrowth and root growth of Lolium perenne L. Grass and Forage Science 52: 401-407.

DONAGHY, D., FULKERSON, W. 1998. Priority of allocation of water soluble carbohydrate reserves during regrowth of Lolium perenne. Grass and Forage Science 53: 211-218.

DONAGHY, D., SCOTT, J., FULKERSON, W. 1997. Effect of defoliation frequency and summer irrigation on survival of perennial (Lolium perenne) and biennial (Lolium multiflorum) ryegrass in the subtropics. Australian Journal of Experimental Agriculture 37: 537-545.

DONAGHY D., FULKERSON, W. 2002. The impact of defoliation frequency and nitrogen fertilizer application in spring on summer survival of perennial ryegrass under grazing in subtropical Australia. Grass and Forage Science 57: 351-359.

DONAGHY, D., TURNER, L., ADAMCZEWSKI, K. 2008. Effect of defoliation management on water-soluble carbohydrate energy reserves, dry matter yields, and herbage quality of tall fescue. Agronomy Journal 100: 122-127.

D’ANGELO, G., POSTULKA, E., FERRARI, L. 2005. Infrequent and intense defoliation benefits dry-matter accumulation and persistence of clipped Arrhenaterum elatius. Grass and Forage Science 60: 17-24.

FULKERSON, W., DONAGHY, D. 2001. Plant-soluble carbohydrate reserves and senescence-key criteria for developing an effective grazing management system for ryegrass-based pastures: a review. Australian Journal of Experimental Agriculture 41: 261-275.

FULKERSON, W., LOWE, K. 2002. Grazing Management. Forages and Pastures. Elsevier Science. Oxford, pp. 11421149.

FULKERSON, W., SLACK, K. 1994. Leaf number as a criterion for determining defoliation time for Lolium perenne. 1. Effect of water soluble carbohydrates and senescence. Grass and Forage Science 49: 373-377.

FULKERSON, W., SLACK, K. 1995. Leaf number as a criterion for determining defoliation time for Lolium perenne: 2.
Effect of defoliation frequency and height. Grass and Forage Science 50: 16-20.

FULKERSON, W., SLACK, K. 2003. Effect of defoliation height and redefoliation interval on regrowth and survival of perennial ryegrass (Lolium perenne) in subtropical dairy pastures. Australian Journal of Experimental Agriculture 43: 121-125.

HUME, D. 1991. Effect of cutting on production and tillering in prairie grass (Bromus willdenowii Kunth) compared with two ryegrass (Lolium) species. 1 Vegetative plants. Annals of Botany 67: 533-541.

NAVE, R., SULC, R., BARKER, D. 2013. Relationships of forage nutritive value to cool-season grass canopy characteristics. Crop Science 53: 341-348

POFF, J., BALOCCHI, O., LOPEZ, I. 2011. Sward and tiller growth dynamics of Lolium perenne L. as affected by defoliation frequency during autumn. Crop and Pasture Science 62: 346-354.

TURNER, L., DONAGHY, D., LANE, P., RAWNSLEY, R. 2006 a. Effect of defoliation management, based on leaf stage, on perennial ryegrass (Lolium perenne L.), prairie grass (Bromus willdenowii Kunth.) and cocksfoot (Dactylis glomerata L.) under dryland conditions. 1. Regrowth, tillering and water soluble carbohydrate concentration. Grass and Forage Science 61: 164-174.

TURNER, L., DONAGHY, D., LANE, P. RAWNSLEY, R. 2006b. Effect of defoliation management, based on leaf stage, on perennial ryegrass (Lolium perenne L.), prairie grass (Bromus willdenowii Kunth.) and cocksfoot (Dactylis glomerata L.) under dryland conditions. 2 Nutritive value. Grass and Forage Science 61: 175- 181.

TURNER, L., DONAGHY, D., LANE, P., RAWNSLEY, R. 2006c. Changes in the physiology and feed quality of prairie grass during regrowth. Agronomy Journal 98: 1326-1332.

VELASCO, M., HERNÁNDEZ, A., GONZÁLEZ, V. 2005. Rendimiento y valor nutritivo del ballico perenne (Lolium perenne L.) en respuesta a la frecuencia de corte. Técnica Pecuaria en México 43: 247-258.

VELASCO, M., HERNÁNDEZ, A., GONZÁLEZ V. 2007. Cambios en los componentes del rendimiento de una pradera de ballico perenne, en respuesta a la frecuencia de corte. Revista Fitotecnia Mexicana 30: 79-87.

WILHELM, W., McMASTER, G. 1995. Importance of the phyllochron in studying development and growth in grasses. Symposium on the phyllochron. Crop Science 35: 1-3. 
